\title{
Flowmetry/ pelvic floor electromyographic findings in patients with detrusor overactivity
}

\author{
Farshid Alizadeh ${ }^{1}$, Shekoufeh Shirani ${ }^{1}$, Mahtab Zargham ${ }^{1}$ \\ ${ }^{1}$ Department of Urology, Isfahan Urology and Kidney Transplantation Research Center, Isfahan University \\ of Medical Sciences, Isfahan, Iran
}

\section{ABSTRACT}

To evaluate different flowmetry/EMG patterns in patients with proven detrusor overactivity (DO) and compare them with that of a group of patients with lower urinary tract symptoms (LUTS) but without DO.

Materials and Methods: We retrospectively evaluated the records of 100 patients with frequent urinary tract infection or any kind of storage or voiding symptoms that had undergone urodynamic testing: 50 cases with proven DO on cystometry who had a good quality flowmetry/EMG and 50 patients without DO. EMG lag time (the time distance between pelvic floor EMG inactivation and the start of urine flow) and different flow curve pattern were recorded and compared.

Results: The age and gender distribution were not statistically significant between the two groups. A negative lag time ( $\leq 0 \mathrm{sec}$ ) and an obstructive pattern were the only parameters that were more commonly seen in the DO group. Sensitivity, specificity, positive predictive value (PPV) and negative predictive value (NPV) of a lag times <2 sec for diagnosing D0 were 70\%, 96\%, 96\% and 72\%, respectively. For a negative lag time, they were 52\%, 100\%, 100\% and 63\%, respectively.

Conclusions: A lag time $<2 \mathrm{sec}$ is a useful flowmetric finding that effectively rules out patients with LUTS that do not have DO (specificity and PPV=96\%). With the cutoff of zero or less, specificity and PPV will be 100\%. It has lower sensitivity and NPV, however, and is not measurable in a considerable population of patients with DO that have concomitant DV.

\section{ARTICLE INFO}

\section{Key words:}

Pelvic Floor; Laser-Doppler

Flowmetry; Lower Urinary Tract

Symptoms

Int Braz J Urol. 2015; 41: 521-6

Submitted for publication:

May 04, 2014

Accepted after revision:

November 08, 2014

\section{INTRODUCTION}

Detrusor overactivity (D0) is the most prevalent type of voiding dysfunction among children. This condition may impose considerable financial burden on the family; not only because of D0 itself, but also due to indirect sequelae of urinary incontinence such as urinary tract infection (UTI) and psychological problems. Prolonged DO may culminate in bladder wall thickening that will affect the function of the bladder in adulthood (1).
Although up to $10 \%$ of healthy neonates and infants may show DO in cystometry and some $60 \%$ of them have uncoordinated voiding, these phenomena will disappear in childhood (2). Persistence of DO may cause symptoms of frequency, urgency, nocturnal enuresis and UTI. When the child tries to suppress the involuntary detrusor contractions by contracting the pelvic floor musculature, he or she may develop dysfunctional voiding, because he or she continues to do so during voluntary micturition, as well. 
The gold standard method for detecting D0 has been urodynamic study (UDS). This method, however, is invasive and harbors the potential risk of inducing UTI.

Recently, some researchers have tried to detect DO and other common voiding conditions, using a non-invasive method of uroflowmetry/ electromyography (EMG) (3-5). The criterion that they have proposed is a short lag time between the start of pelvic floor relaxation (determined by pelvic floor EMG inactivation) and the start of urine flow during voluntary voiding.

In this study, we evaluated the flowmetric patterns that were observed in a cohort of patients with detrusor overactivity to see if there are other findings than a short lag time in these patients and compared them with patients without DO in order to determine which are more prevalent in DO patients. With the presence of a control group, it was also possible to calculate the sensitivity, specificity and predictive values of a short lag time.

\section{MATERIALS AND METHODS}

From Oct 2009 to Sep 2013, 1372 urodynamic tests were performed at our center. With institutional review board approval, we retrospectively evaluated these tests and enrolled 100 of them into the study: 50 cases with proven DO on cystometry who had a good quality flowmetry-EMG (i.e. voided volume $\geq 1 / 2$ estimated bladder capacity (EBC) in children and $150 \mathrm{~mL}$ in adults, without EMG artefact) and 50 cases without DO on cystometry as the control group.

Indications for performing UDS in patients who later proved to have no DO included prolonged enuresis, urinary incontinence in women and high grade vesicoureteral reflux in children who were candidates for surgery, recurrent UTI and refractory lower urinary tract symptoms (LUTS).

All tests were performed on a Laborie $^{\circledR}$ urodynamic system (Laborie Medical Technologies, Toronto, Ontario, Canada), using double lumen urethral catheters $(6 \mathrm{~F}$ in children and $8 \mathrm{~F}$ in adults) and rectal balloons, according to International Continence Society recommendations (6). Tests were performed in sitting position and patients with a known history of spinal cord injury, spinal dysraphism, intervertebral disk herniation, head trauma, stroke and central nervous system tumors were excluded.

None of the patients in either group had a gross anomaly in the external genitalia. Bladder and urethral anomalies in children with a history of UTI was evaluated by a micturition cystourethrography. Surgically correctable urethral anomalies were managed first and UDS was performed in patients with recurrent UTI or unresolved storage or voiding symptoms.

In young male adults with voiding symptoms, a retrograde urethrography was performed first. In middle aged and older males, treatment for presumed prostatic hyperplasia was initiated and if no response was observed, UDS was performed.

We used an EMG module with a high sampling rate and surface electrodes were placed at 3 and 9 o'clock positions at the margin of anal verge. A sample flowmetry/EMG in a patient with documented DO, showing a negative lag time is presented in Figure-1. All patients were off anticholinergics for at least 12 weeks prior to the test.

Real time bladder ultrasound was performed just before and after the test to measure the urine volume and post-void residual urine volume (PVR). In children, Koff formula was used for calculating EBC: (age in years+2) x30 $\mathrm{mL}$ up to $350 \mathrm{~mL}$.

Patients who had a bell-shaped flow pattern, a quiet pelvic floor and an EMG lag time between 2-6 seconds were considered completely normal. If the EMG became inactive $0-2$ seconds before the initiation of flow, we considered it as a short lag time and if inactivation occurred after that, it was defined as negative lag time. Bladder neck dysfunction was defined as a prolonged lag time ( $>6 \mathrm{sec}$ ), a depressed curved with a right shift and silent pelvic floor. Patients who had active EMG during voiding were considered to have dysfunctional voiding (DV), whether the flow curve was bell-shaped or staccato. Obstructive pattern was defined as a plateau curve with lag time $<6$ sec.

Data were entered into SPSS version 22 and Student's t test, Fisher's exact test and chi-square test were used for analyzing data, with significance considered at $\mathrm{p}<0.05$. 
Figure 1 - A sample of flowmetry/EMG in a patient with documented DO, showing a negative lag time.

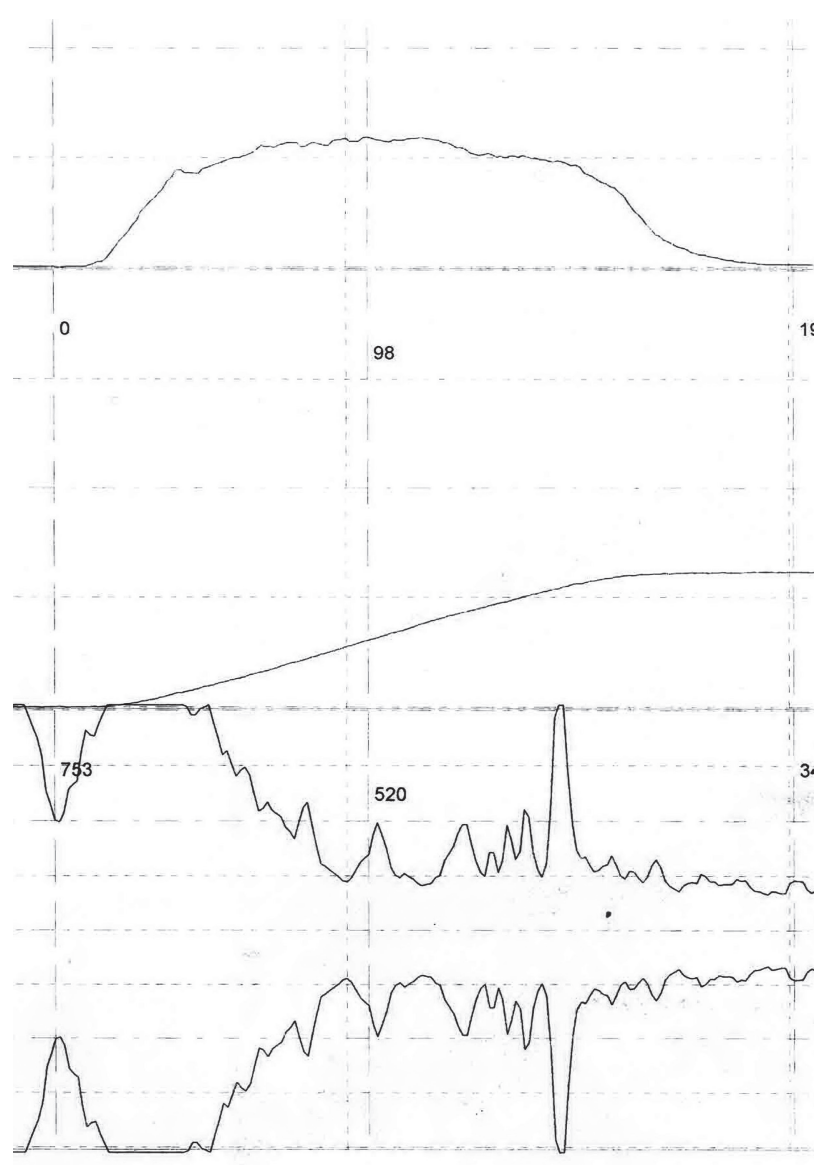

\section{RESULTS}

Twenty-three patients (46\%) in the case group and 17 patients (34\%) in the control group were male. The gender distribution between the two groups was not statistically significant $(\mathrm{P}=0.32)$.The mean patients' age in the $\mathrm{DO}$ and control groups were $27.8 \pm 22.9$ years and $32 \pm 19$ years, respectively; the difference was not significant $(\mathrm{P}=0.56)$. Figure- 2 shows the age distribution of patients and controls.

Flowmetric findings are summarized in Table-1. Significantly more patients in the control group had a normal curve and normal lag time. On the other hand, a negative lag time and an obstructive pattern were more commonly seen in the D0 group. Other findings were not significantly different between the two groups.

In the DO group, one patient with obstructive pattern and one with tower-shaped flow curve had a normal lag time. The "super-voider" patient in the control group had also a normal lag time.

The most common abnormal finding in the D0 group was a negative lag time (36\%); while in the control group, it was DV (46\%).

If we exclude patients with DV, in whom a lag time cannot be measured, in the DO group $23 \%$ had a normal lag time, 18\% a short, and 53\%

Figure 2 - Age distribution of patients with and without DO.

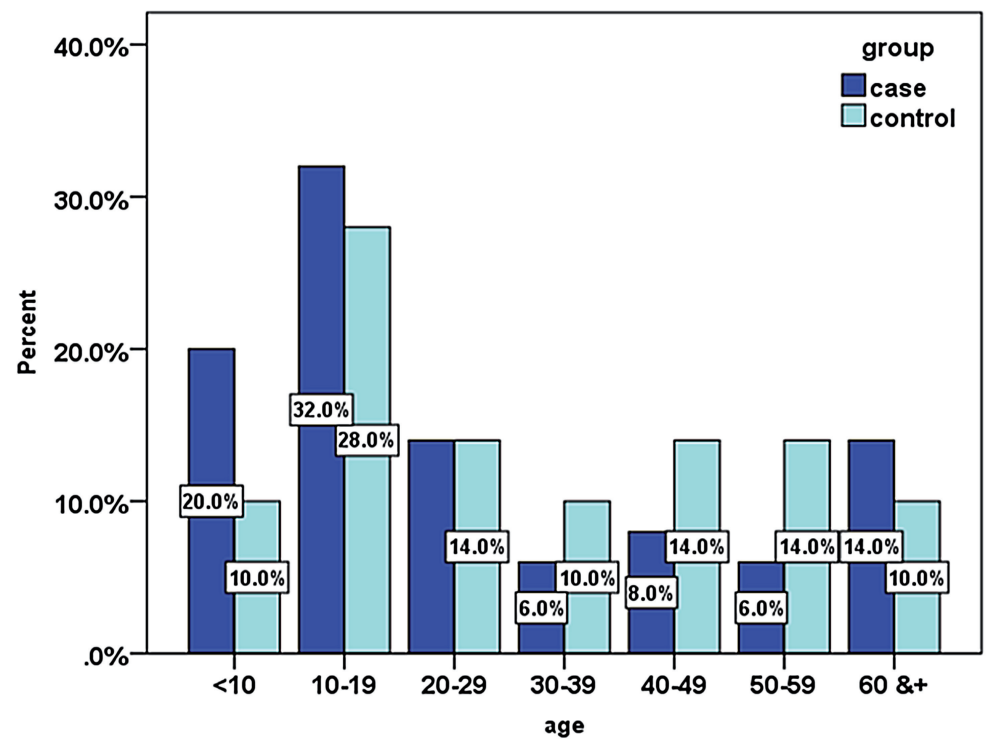


Table 1 - Flowmetric findings in DO patients and controls. DO: detrusor overactivity, BND: bladder neck dysfunction, DV: dysfunctional voiding.

\begin{tabular}{lccc}
\hline & DO & Control & \\
\hline Flowmetric finding & Number (\%) & Number (\%) & P value \\
\hline Completely normal curve & $6(12)$ & $23(46)$ & $<0.001$ \\
Normal lag time (2-6 seconds) & $8(16)$ & $24(48)$ & 0.001 \\
Short lag time (0-2 seconds) & $6(12)$ & $1(2)$ & 0.11 \\
Negative lag time & $18(36)$ & $0(0)$ & $<0.001$ \\
$\quad$ BND & $2(4)$ & $2(4)$ & 1 \\
$\quad 16(32)$ & $23(46)$ & 0.15 \\
DV & $6(12)$ & $0(0)$ & 0.027 \\
Obstructive pattern & $2(4)$ & $1(2)$ & 0.99 \\
\hline
\end{tabular}

a negative lag time. These figures were 89\%, 4\% and $0 \%$ in the control group, respectively. The difference was still significant for normal and negative lag times $(p<0.001)$ and insignificant for short lag time $(\mathrm{p}=0.12)$.

The remaining patients had BND with a long lag time (6\% in the DO and 7\% in the control group; $\mathrm{p}=0.99$ ).

Sensitivity, specificity, positive predictive value (PPV) and negative predictive value (NPV) for lag times $<2$ sec and $\leq 0$ sec are presented in Table- 2 .

\section{DISCUSSION}

Overactive bladder $(\mathrm{OAB})$ is a syndrome of symptoms, with its hallmark being urgency. However, not all patients with urgency have D0; patients with DV and BND frequently complain of urgency while the primary treatment for them is not anticholinergics (5). Van Batavia et al. evaluated a group of children with LUTS by flowmetry/EMG and found that the most common finding in patients with DV was day time incontinence, while they were reported frequency and urgency in patients with DO and BND (4). These finding suggest that irritative LUTS are the most common complaint in patients with DO, DV and BND. Therefore, it is reasonable to tailor the treatment according to the underlying condition rather than prescribing anticholinergics for all patients with urgency.

Attempts have been made to diagnose different types of bladder dysfunction by the non-invasive method of flowmetry. A tower-shaped flow curve has been attributed to D0 (7). This finding was seen in two patients (4\%) with and one patient (2\%) without DO. We found it a weak indicator of D0 ( $\mathrm{p}=0.99)$. Van Batavia et al. reported a tower flow pattern in 10\% of their patients, all with a short or negative lag time (4), while one of our DO patients had a normal lag time.

Table 2 - Sensitivity, specificity, positive predictive value (PPV) and negative predictive value (NPV) for lag times<2 sec and $\leq \mathbf{0}$ sec.

\begin{tabular}{lcccc}
\hline & Sensitivity $(\%)$ & Specificity $(\%)$ & PPV $(\%)$ & NPV $(\%)$ \\
\hline Lag time $<2 \mathrm{sec}$ & 70 & 96 & 96 & 72 \\
Lag time $\leq 0 \mathrm{sec}$ & 52 & 100 & 100 & 63 \\
\hline
\end{tabular}


A short EMG lag time is another indicator of DO. Combs et al. found a positive predictive value (PPV) and specificity of 100\% for a zero or negative lag time. The sensitivity was $88 \%, 80 \%$ and $70 \%$ for lag times of $<2 \mathrm{sec},<1 \mathrm{sec}$ and $\leq 0$, respectively (5). They argue that since the bladder volume at the time of voiding may not be high enough to provoke D0, a short or negative lag time is not present in all patients with DO. Besides, a patient may be able to voluntarily abort the D0 just prior to volitional voiding. These authors believe that repeating the study may increase the sensitivity. In our study specificity and PPV were the same; although sensitivity was lower (for lag time <2 sec: $70 \%$ vs. $88 \%$ and for lag time $\leq 0$, $52 \%$ vs. $70 \%$ ). Instead of lag time $\leq 0 \mathrm{sec}$, if we consider lag time $<2 \mathrm{sec}$ as abnormal, then the sensitivity will increase from $52 \%$ to $70 \%$ without sacrificing much of specificity.

In the present study, although a short lag time $(0-2 \mathrm{sec})$ was more common in the DO group (6 vs. 1 patients), the difference was not significant $(\mathrm{p}=0.11)$; this could be because of the small sample size.

DV was a common finding in both groups. In these patients, the flow curve may or may not have a staccato pattern. However, EMG is always active during voiding and a lag time cannot be measured. A short lag time does not apply to patients with BND either, in whom lag time is longer than normal. These two groups formed more than one-thirds of patients in the DO group. Therefore, there are substantial numbers of patients with DO, in whom a short lag time is not applicable.

Our study has several limitations:

1. It has a retrospective design.

2. Patients' symptoms have not been recorded to be correlated with flowmetric findings.

3. Flowmetry is not repeated after treatment to check for its change in pattern.

4. Our control group consisted of patients with some kind of LUTS without proven DO on UDS and not completely normal people. Some of them might have had DO that was not evident on UDS.
5. Previous studies that evaluated the lag time were performed on children only, while our study population consisted of children and adults.

6. The sample size was relatively small.

7. In spite of the previous studies that have evaluated only pediatric patients, our study group was heterogeneous in terms of patients' age. Considering the differences in the neural system function that might be present between children and adults, more studies are needed to confirm the reliability of a short lag time in adults and the elderly.

\section{CONCLUSIONS}

A negative lag time and an obstructive pattern were significantly more common in the D0 group while a completely normal curve and a normal lag time were more prevalent in the control patients. The prevalence of a short lag time, BND, DV and a tower-shaped flow curve was not significantly different between the two groups.

A lag time $<2$ sec is a useful flowmetric finding that effectively rules out patients with LUTS that do not have DO (specificity and PPV=96\%). With the cutoff of zero or less, specificity and PPV will be 100\%. It has lower sensitivity and NPV, however, and is not measurable in a considerable population of patients with DO that have concomitant DV.

Further studies are recommended in separate groups of pediatric, adult, elderly and neurologically impaired patients to confirm these findings in more homogenous groups.

\section{REFERENCES}

1. Franco I. Pediatric overactive bladder syndrome: pathophysiology and management. Paediatr Drugs. 2007:9:379-90.

2. Nevéus T, Sillén U. Lower urinary tract function in childhood; normal development and common functional disturbances. Acta Physiol (Oxf). 2013;207:85-92.

3. Glassberg KI, Combs AJ, Horowitz M. Nonneurogenic voiding disorders in children and adolescents: clinical and videourodynamic findings in 4 specific conditions. J Urol. 2010;184:2123-7. 
4. Van Batavia JP, Combs AJ, Hyun G, Bayer A, MedinaKreppein D, Schlussel RN, et al. Simplifying the diagnosis of 4 common voiding conditions using uroflow/ electromyography, electromyography lag time and voiding history. J Urol. 2011;186:1721-6.

5. Combs AJ, Van Batavia JP, Horowitz M, Glassberg KI. Short pelvic floor electromyographic lag time: a novel noninvasive approach to document detrusor overactivity in children with lower urinary tract symptoms. J Urol. 2013;189:2282-6.
6. Abrams P, Cardozo L, Fall M, Griffiths D, Rosier P, Ulmsten $\mathrm{U}$, et al. Standardisation Sub-Committee of the International Continence Society. The standardisation of terminology in lower urinary tract function: report from the standardisation sub-committee of the International Continence Society. Urology. 2003;61:37-49.

7. Roihuvuo-Leskinen HM, Koskimäki JE, Tammela TL, LahdesVasama TT. Urine flow curve shapes in adults with earlier vesicoureteral reflux. Eur Urol. 2008;54:188-94.

Correspondence address:

Farshid Alizadeh, MD

Flat number 10, No. 22, 16th alley Shams Abadi St., Isfahan, Iran

Fax: + $98311235-0532$

E-mail: f_alizadeh@med.mui.ac.ir 